\title{
THE EFFECT OF RECOMBINATION ON THE PRIMARY PHOTO-ELECTRIC CURRENT FROM A CRYSTAL
}

\author{
By W. Howard Wise
}

\begin{abstract}
Taking into account recombination, mathematical expressions are derived for the primary photo-electric current to be expected from crystals both when the light is normal to the electric field and when the light is parallel and opposite to the electric field. On comparing with the experiments on rock salt it is found that recombination is sufficient to account for the drop in current with time. The decrease in current when the light is opposite to the field and most strongly absorbed, is shown to be partially accounted for by recombination. The effect of the unknown process causing the major portion of the drop is thus isolated and a more rigorous test of any theory which may be proposed to account for it made possible.
\end{abstract}

\section{I}

M

ANY crystals when placed in an electric field and illuminated by light of a frequency range peculiar to the crystal are found to be conducting. The experiments of Gudden and Pohl ${ }^{1}$ on this subject show that the current so obtained is of two kinds: (1) a primary current due to photo-electrons which the light has liberated from the atoms of the crystals, (2) a secondary current representing the motion of the positive ions toward the cathode. Under suitable experimental conditions the current is entirely of the first or primary kind. In this case the positive ions accumulate in the crystal and by capturing electrons on their way to the anode materially reduce the primary current. $^{2}$ It is the purpose of this investigation to calculate, with certain idealizations, how much this recombination reduces the primary current.

The experiments are of two classes; in the first the light is perpendicular to the electric field (fig. 3); in the second the light is parallel and opposite to the electric field ${ }^{3}$ (Fig. 6).

If the light is parallel to the electric field lateral diffusion of the electrons can have no effect on the current because the field is normal to layers of electrons of constant density.

If the light is perpendicular to the electric field the effect of lateral diffusion is to flatten out the original density distribution $e^{-\mu x}$ given

\footnotetext{
${ }^{1}$ Gudden and Pohl, Zeits. f. Physik 17, 331 (1923).

2 Gudden and Pohl, Naturwissenschaften 11, 354 (1923).

${ }^{3}$ Gudden and Pohl, Phys. Zeits. 26, 481 (1925).
} 
by the light. Those electrons diffusing from regions of greater electron density (and consequent greater ion density) to regions of lesser electron density (and consequent lesser ion density) have a better chance of escaping recombination with some ion and reaching the anode than those electrons which do not diffuse. On the other hand those electrons which do not diffuse have a smaller chance of reaching the anode than they would have, did not some others diffuse, because they must now escape not only the ions normally in their path but also those ions which the diffusing electrons escape. The effect of diffusion is thus somewhat self-compensating and a considerable change in the distribution of electrons from this cause need not, so far as can be seen without actually taking it into account in the calculations, cause a material change in the primary current. As it is not an easy matter to take diffusion into account in calculating the current to be expected

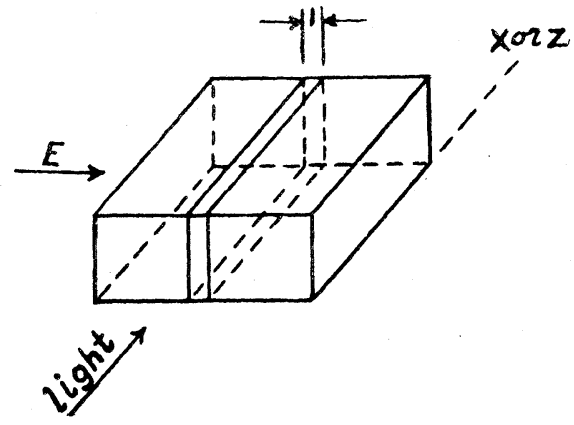

Fig. 1.

it will be shown instead, that, for the experiments to which the expression derived for the current is to be applied, diffusion does not sensibly alter an original distribution while it traverses the crystal. This is done in section II. In section III the primary current is computed for the case of a field at right angles to the incident light and in section IV it is computed for the case of a field parallel and opposite to the incident light.

\section{II}

Let free electrons be present in a crystal and be distributed according to the law $e^{-\mu x}$ which is the law by which they are photo-electrically liberated. $\mu$ is the absorption constant for the light used. Suppose now that these electrons are uniformly distributed in a unit layer under the curve $e^{-\mu x}$. The problem before us is to find out how much diffusion has flattened out this original distribution by the time the field $E$ has pulled the unit layer over to the anode. 
Because of diffusion each element of volume $e^{-\mu x} d x$ in the unit layer will in time assume the distribution $k e^{-a u 2}$ where $k$ and $a$ are determined by the condition

$$
\begin{aligned}
k \int_{-\infty}^{\infty} e^{-a{ }^{2}} d u & =\text { area under the elementary probability curve } \\
& =k \sqrt{\pi / a}=e^{-\mu x} d x
\end{aligned}
$$

Since an electron can not diffuse from the surface of the crystal into the air the probability curve must be folded back at the surface of the crystal. The contribution of an element of volume $e^{-\mu x} d x$ to the electrons at any other point in the same unit layer after the redistribution is thus from two parts of the little probability curve

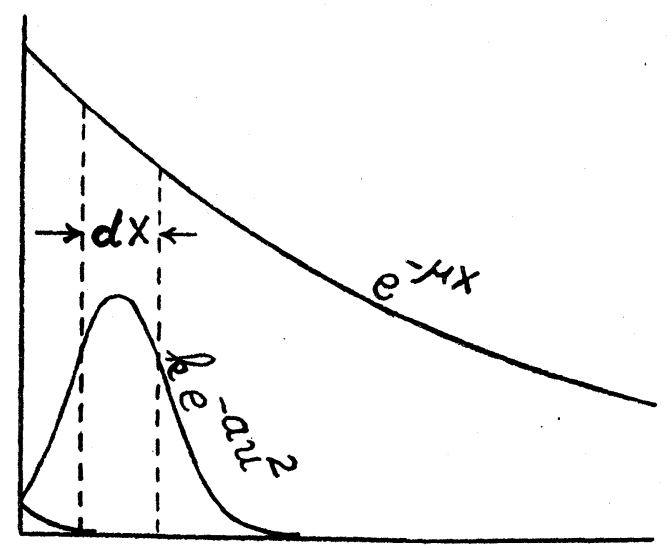

Fig. 2.

distant $2 x$ apart. The number of electrons at a point $z$ due to the diffusion of $e^{-\mu x} d x$ electrons starting from the point $x$ is therefore

$$
d \rho=k e^{-a(x-z)^{2}}+k e^{-a(x+z)^{2}}
$$

But from (1) we have $k=\sqrt{a / \pi} e^{-\mu x} d x$ and consequently

$$
d \rho=\sqrt{a / \pi}\left\{e^{-a(x-z)^{2}}+e^{-a(x+z)^{2}}\right\} e^{-\mu x} d x
$$

The total number of electrons between $z$ and $z+1$ is then

$$
\rho=\sqrt{a / \pi} \int_{0}^{\infty}\left\{e^{-a(x-z)^{2}}+e^{-a(x+z)^{2}}\right\} e^{-\mu x} d x
$$

This new distribution function $\rho$ satisfies the differential equation

$$
\frac{d^{2} \rho}{d z^{2}}=\mu^{2} \rho-2 \mu \sqrt{a / \pi} e^{-a z 2}
$$


The properties of $\rho$ are not apparent from (2) but (3) shows that $\rho$ does have the characteristics to be expected from the nature of the problem: for if $a \doteq \infty$, meaning that there is no diffusion, (3) reduces to $d^{2} \rho / d z^{2}=\mu^{2} \rho$ of which the solution is $\rho=e^{-\mu z}$ which is the original distribution law; as $z$ becomes very large (3) again reduces to $d^{2} \rho / d z^{2}=$ $\mu^{2} \rho$ with the solution $\rho=e^{-\mu z}$ which means that if the original distribution is quite uniform, as it is for large values of $z$, then diffusion does not affect it.

The expression (2) has yet to be put in a form suitable for computation.

$$
\begin{gathered}
\rho=\sqrt{a / \pi} \int_{0}^{\infty}\left\{e^{-a(x-z)^{2}}+e^{-a(x+z)^{2}}\right\} e^{-\mu x} d x \\
=e^{\mu^{2} / 4 a}\left[\cosh \mu z-\sqrt{a / \pi}\left(e^{\mu z} \int_{0}^{z+\mu / 2 a} e^{-a s^{2}} d S-e^{-\mu z} \int_{0}^{z-\mu / 2 a} e^{-a s^{2}} d S\right)\right]
\end{gathered}
$$

If the electron can be looked upon as having a mean free path $l$ then

$$
a=4^{2} / \pi^{2} n l^{2},
$$

where $n$ is the number of collisions it has made,

$$
\begin{aligned}
& \mu=e l / 2 m v=\text { mobility of the electron } \\
& V=\mu E=e E l / 2 m v=\text { velocity with which it proceeds through crystal, } \\
& V / v=e E l / 2 m v^{2}=\text { ratio of forward progress to total random motion, } \\
& n=v d / V l=\frac{2 d}{e E l^{2}}=m v^{2}=\begin{array}{c}
\text { number of collisions made in advancing } \\
\text { a distance } d \text {. }
\end{array} \\
& \therefore a=4^{2} / \pi^{2} n l^{2}=\frac{4^{2}}{\pi^{2}} \frac{e E}{4 d} \frac{1}{\frac{1}{2} m v^{2}}=3130 E_{v} / T d
\end{aligned}
$$

where $E_{v}$ is the electric field in volts per $\mathrm{cm}$ and $T$ is the absolute temperature. Let $T=306^{\circ}$; take $d=15 \mathrm{~cm}$ or half the crystal thickness ; assume $E_{v}=117.5$; then $a=8100$.

As $\mu$ affects only the abscissa scale when $e^{-\mu x}$ is plotted let $\mu=1$.

$$
\begin{aligned}
\rho=e^{1 / 32,400}\left[\cosh z-\frac{90}{\sqrt{ } \pi}\left(e^{z} \int_{0}^{z+1 / 16200}\right.\right. & e^{-a s^{2}} d s \\
& \left.\left.-e^{-z} \int_{0}^{z-1 / 16200} e^{-a s^{2}} d s\right)\right]
\end{aligned}
$$

Let $90 s=t$ and then if $s=z \pm 1 / 16200 \quad t=90 z \pm 1 / 180$

$$
\rho=\cosh z-\frac{1}{\sqrt{ } \pi}\left(e^{z} \int_{0}^{90 z+1 / 180} e^{-t^{2}} d t-e^{-z} \int_{0}^{90 z-1 / 180} e^{-t^{2}} d t\right)
$$


The greatest change due to diffusion must occur at the origin $z=0$ for it is here that the slope of the curve $e^{-\mu x}$ is greatest ( $x$ positive). Placing therefore $z=0$ we calculate

$$
\rho_{0}=1-\frac{1}{\sqrt{ } \pi} \int_{1 / 180}^{1 / 180} e^{-t^{2}} d t=1-.0062=.9938,
$$

or that the average maximum change in an ordinate, under the conditions stated is 0.6 percent or less; less because the average electron travels less than half the crystal thickness.

From these considerations it is seen to be permissible to assume in the mathematical work of deriving the expression for the primary. current that the electrons move only in the direction of the electric field.

\section{III}

It is assumed that an electron reaches the anode unless it recombines with an ion and that the radiation emitted when recombination occurs does not liberate another electron before it reaches the surface of the crystal and escapes. Were this condition not completely fulfilled the effect would merely be that diffusion and the distance between ions would both appear to be greater than normal: for the disappearance of an electron in one place and its appearance in another could be looked upon as diffusion and by this process one collision with an ion has been lost.

The mean free path of the electrons, referred to ions, is taken to be $\lambda=1 / N \pi \sigma^{2} b$ where $N$ is the number of ions per cc, $\sigma$ is the radius of an ion and $b$ may be a function of the mobility of the electrons, of the field, and consequently a function of the concentration of ions but is assumed to be a constant. This point will be brought up again.

If $\lambda(s)$, the mean free path of electrons appropriate to the position $s$, be so defined that the chance of an electron's recombining in the distance $d s$ is $d s / \lambda(s)$, it may readily be shown that the chance $P\left(s_{1}, s_{2}\right)$ of an electron's going from a position $s_{1}$ to a position $s_{2}$ without recombining is given by

$$
P\left(s_{1}, s_{2}\right)=e^{-\int_{s_{1}}^{s_{2}} d s / \lambda(s)}
$$

Then the chance that an electron liberated a distance $z$ from the cathode at time $t$ will go to a point distant $y$ from the cathode and recombine in the distance $d y$ is $P(z, y, t) d y / \lambda(y, t)$ provided we may 
neglect the change in $\lambda$ at any point during the time it takes an electron to go from $z$ to $y$. If this is not permissible we must write instead that the chance is $P\left(z, y, t_{r}\right) d y / \lambda(y, t)$ where

$$
P\left(z, y, t_{r}\right)=e^{-\int_{z}^{y} \frac{d s}{\lambda[s, t-(y-s) / \mu E]}}
$$

and $\mu$ is the mobility or velocity in a unit field.

The rate at which electrons are being liberated is a function of $x$ only, $F(x)$. Then from the element of volume $k d x d z$ there are liberated $k d x d z \cdot F(x)$ electrons per sec. of which the number recombining in the element of volume $k d x d y$ is $k d x d z \cdot F(x) P\left(z, y, t_{r}\right) d y / \lambda(y, t)$ at the time $t$ if $t \geqq(y-z) / \mu E$.

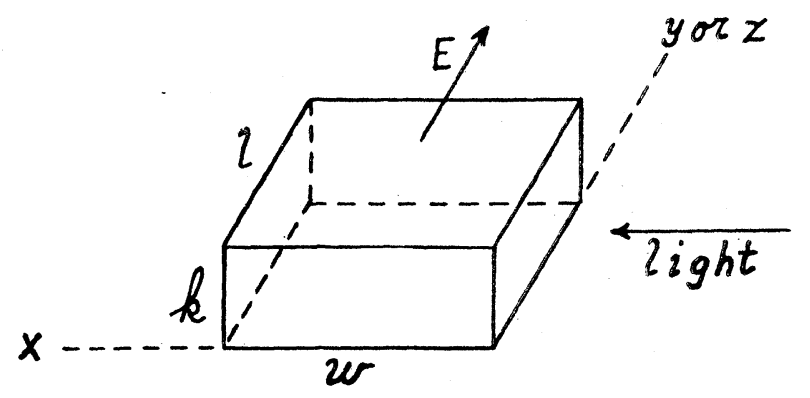

Fig. 3.

If there are $n$ electrons per sq. $\mathrm{cm}$ per sec. leaving the cathode, the number of these which combine with ions in the element of volume $k d x d y$ is $k d x \cdot n P\left(0, y, t_{r}\right) d y / \lambda(y, t)$ per sec. at the time $t$. In the experiments to which this work is to be applied $n=0$ but it will be retained for the present.

During the time $t<l / \mu E$ the total number of liberated electrons which recombine in $k d x d y$ is

$$
k d x d y F(x) \frac{1}{\lambda(y, t)} \int_{y-\mu E t}^{y} P\left(z, y, t_{r}\right) d z
$$

per sec.

So up to the time $t=y / \mu E$ the total number of electrons recombining per sec. in $k d x d y$ is

$$
k d x d y 1 / \lambda(y, t)\left[n P\left(y-\mu E t, y, t_{r}\right)+F(x) \int_{y-\mu E t}^{y} P\left(z, y, t_{r}\right) d z\right]
$$


while the number being produced per sec. is $k d x d y F(x)$. The difference must be the net rate of increase of ions which is

$$
k d x d y \frac{\partial N}{\partial t}=k d x d y \frac{1}{\pi \sigma^{2} b} \frac{\partial}{\partial t} \frac{1}{\lambda(y, t)}
$$

and hence we have

$\frac{1}{\pi \sigma^{2} b} \frac{\partial}{\partial t} \frac{1}{\lambda}=F(x)-\frac{1}{\lambda}\left[n P\left(y-\mu E t, y, t_{r}\right)+F(x) \int_{y-\mu E t}^{y} P\left(z, y, t_{r}\right) d z\right]$

as the defining equation for $\lambda=\lambda(x, y, t)$ up to $t=y / \mu E$. The second term on the right has the lower limit $y-\mu E t$ for the integral because the chance that the $n k d x$ electrons reach the point $y-\mu E t$ is 1 if $t<y / \mu E$. If $t>y / \mu E$ we must write instead of (1)

$\frac{1}{\pi \sigma^{2} b} \frac{\partial}{\partial t} \frac{1}{\lambda}=F(x)-\frac{1}{\lambda}\left[n P\left(0, y, t_{r}\right)+F(x) \int_{0}^{y} P\left(z, y, t_{r}\right) d z\right]$

It is convenient to rewrite Eq. (1) as

$$
A \frac{\partial}{\partial t} \frac{1}{\lambda}=1-\frac{1}{\lambda}\left[B P\left(y-\mu E t, y, t_{r}\right) \int_{y-\mu E t}^{y} P\left(z, y, t_{r}\right) d z\right]
$$

where $A=\left[\pi \sigma^{2} b F(x)\right]^{-1}$ and $B=n / F(x)$

or $\quad-A \frac{\partial}{\partial t} \log \lambda=\lambda-\left[B P\left(y-\mu E t, y, t_{r}\right)+\int_{y-\mu}^{y} E t\left(z, y, t_{r}\right) d z\right]$

Now let

$$
\lambda=1 / \frac{\partial}{\partial s} \log v\left(s, t-\frac{y-s}{\mu E}\right)
$$

under the integral signs and

$$
1 / \frac{\partial}{\partial y} \log v(y, t)
$$

outside the integral signs where $s=y$. Since $\lambda=\infty$ if $t=0 v(y, 0=$ $v_{0}=$ const. With this change of variable Eq. (1.2) becomes

$$
A\left(\frac{\partial v}{\partial t}-v \frac{\partial^{2} v}{\partial y \partial t} / \frac{\partial v}{\partial y}\right)+v^{2} / \frac{\partial v}{\partial y}=B v_{0}+\int_{y-\mu E t}^{y} v\left(z, t-\frac{y-z}{\mu E}\right) d z
$$


If we differentiate (2) with respect to $y$ and $t$ and then add the results we get the following differential equation

$$
\begin{aligned}
\frac{\partial}{\partial y}\left[A \left(\frac{\partial v}{\partial t}-v \frac{\partial^{2} v}{\partial y \partial t} /\right.\right. & \left.\left.\frac{\partial v}{\partial y}\right)+v^{2} / \frac{\partial v}{\partial y}\right] \\
& +\frac{1}{\mu E} \frac{\partial}{\partial t}\left[A\left(\frac{\partial v}{\partial t}-v \frac{\partial^{2} v}{\partial y \partial t} / \frac{\partial v}{\partial y}\right)+v^{2} / \frac{\partial v}{\partial y}\right]=v
\end{aligned}
$$

which must be solved for $v$ if we are to know how the current rises when the light in turned on.

The current passing any point. The part of the $k d x d z F(x)$ electrons liberated per second from the element of volume $k d x d z$ that reaches the point $y$ is

$$
k d x d z F(x) \quad P\left(z, y, t_{r}\right)
$$

Of the $n$ electrons passing $1 \mathrm{sq} . \mathrm{cm}$ per sec. at the point $y-\mu E t$ at the time $t=0$ the number passing $1 \mathrm{sq}$. $\mathrm{cm}$ per sec. at the point $y$ at the time $t$ is

$$
n P\left(y-\mu E t, y, t_{r}\right)
$$

up to the time $t=y / \mu E$.

Then the total number of electrons passing 1 sq. cm per sec. at time $t$ at the point $y$ is

$$
\delta I_{y} / e=M=F(x) \int_{y-\mu E t}^{y} P\left(z, y, t_{r}\right) d z+n P\left(y-\mu E t, y, t_{r}\right)
$$

Placing now

$$
\lambda=1 / \frac{\partial}{\partial s} \log v\left(s, t-\frac{y-s}{\mu E}\right)
$$

as before we get

or

$$
\begin{gathered}
M=F(x)\left[B v_{0} / v+\frac{1}{v} \int_{y-\mu E t}^{y} v\left(z, t-\frac{y-z}{\mu E}\right) d z\right] \\
M=\frac{F}{v}\left[A\left(\frac{\partial v}{\partial t}-v \frac{\partial^{2} v}{\partial y \partial t} / \frac{\partial v}{\partial y}\right)+v^{2} / \frac{\partial v}{\partial y}\right]
\end{gathered}
$$

From $^{-}(3)$ then we have

or

$$
\frac{\partial}{\partial y}\left(\frac{M v}{F}\right)+\frac{1}{\mu E} \frac{\partial}{\partial t}\left(\frac{M v}{F}\right)=v
$$

$$
\frac{\partial^{2}}{\partial y^{2}}\left(\frac{M v}{F}\right)-\frac{\partial v}{\partial y}=\frac{1}{\mu^{2} E^{2}} \frac{\partial^{2}}{\partial t^{2}}\left(\frac{M v}{F}\right)-\frac{1}{\mu E} \frac{\partial v}{\partial t}
$$


In all the experiments of Gudden and Pohl the current leaps immediately to a maximum and then decreases. This indicates that $\mu E$ is very large and consequently that the time taken for an electron to cross the crystal is small compared with the time required for the distribution of ions to change sensibly. We have then to deal with (1.1) and with (3) rewritten as

or

$$
\frac{\partial}{\partial y}\left[A\left(\frac{\partial v}{\partial y}-v \frac{\partial^{2} v}{\partial y \partial t} / \frac{\partial v}{\partial y}\right)+v^{2} / \frac{\partial v}{\partial y}\right]=v
$$

$$
A \frac{\partial^{2} v}{\partial y \partial t}-v-\frac{\partial}{\partial y}\left[v\left(A \frac{\partial^{2} v}{\partial y \partial t}-v\right) / \frac{\partial v}{\partial y}\right]=0
$$

or $\partial^{2} v / \partial y \partial \tau=v \quad$ where $\tau=t / A=\pi \sigma^{2} b F(x) t$

Following the suggestion of (5) that $t$ and $y$ may enter into $v$ in the same way, we assume $v=v[(\tau+g)(y+h)]$ and find that (3.3) reduces to the total differential equation (the other simple and most obvious assumption $v=v(\tau+y)$ does not yield a suitable form of solution $):(\tau+g)(y+h) v^{\prime \prime}+v^{\prime}=v$. Letting $(\tau+g)(y+h)=-\alpha^{2} / 4$ this becomes

$$
v^{\prime \prime}+v^{\prime} / \alpha+v=0
$$

and therefore

$$
v=J_{0}(\alpha)=J_{0}[2 i \sqrt{(\tau+g)(y+h)}]
$$

Using now the fact that $n=0$ and $\mu E$ is large we may rewrite (2.2) in the following form

$$
A \frac{\partial}{\partial t} \frac{1}{\lambda}=1-\frac{1}{\lambda} \int_{0}^{y} P(z, y, t) d z
$$

or

$$
\frac{\partial v}{\partial \tau}=\int_{0}^{y} v(z, \tau) d z
$$

where we have used (3.3) and $1 / \lambda=\partial \log v / \partial y$.

The condition

$$
\lambda=1 / \frac{\partial}{\partial y} \log v=\infty
$$

if $t=0$ makes $g=0$ for only then will $v$ be independent of $y$ when $t=0$. Substituting $J_{0}[2 i \sqrt{\tau(y+h)}]$ into (2.6) we find that $h=0$. Therefore

$$
\lambda=1 / \frac{\partial}{\partial y} \log J_{0}(2 i \sqrt{\tau y})
$$


Substituting (3.3) in (4.1) we find

Hence

$$
\delta I_{y}=e F \frac{\partial v}{\partial \tau} / v=\frac{e}{\pi \sigma^{2} b v} \frac{\partial v}{\partial t}=\frac{e y F}{v} \frac{\partial v}{\partial(\tau y)}=e y F v^{\prime} / v
$$

$$
I_{l}=e k l \int_{0}^{w} \frac{v^{\prime}(\tau l)}{v(\tau l)} F(x) d x
$$

Wherein we have taken account of the fact that the crystal is $k \mathrm{~cm}$ high and that the current is measured at the anode where $y=l$. The ratio $v^{\prime} / v$ may be expanded into a power series and then

$$
I=e k l \int_{0}^{w}\left[1+a_{1} \tau l+a_{2}(\tau l)^{2}+a_{3}(\tau l)^{3}+\cdots\right] F(x) d x
$$

If $Q_{0}$ ergs of energy enter $1 \mathrm{sq}$. $\mathrm{cm}$. of the surface each second the energy remaining in this square beam at a depth $x$ in the crystal is $Q_{1}=Q_{0} e^{-\mu x}$. Of the energy remaining in the beam when it reaches the rear face a fraction $r$ is reflected and this fraction behaves just like the original beam. The energy at any point is thus made up of an infinite series of terms. The sum, $Q$, of this series is

$$
Q_{0}\left(e^{-\mu x}+r e^{-2 \mu w} e^{\mu x}\right) /\left(1-r^{2} e^{-2 \mu w}\right)=Q_{0} A \cosh \{\mu(x-w)+\log \sqrt{\gamma}\}
$$

where

$$
A=2 \sqrt{r} e^{-\mu w} /\left(1-r^{2} e^{-2 \mu w}\right)
$$

The rate at which electrons are being liberated at the depth $x$ is

$$
F(x)=-\frac{1}{h \nu} \frac{d Q}{d x}=-\left(Q_{0} \mu / h \nu\right) A \sinh [\mu(x-w)+\log \sqrt{r}]
$$

therefore

$$
\begin{aligned}
I & =e k l \int_{0}^{w}\left[1+a_{1}\left(\pi \sigma^{2} b t l\right) F+a_{2}\left(\pi \sigma^{2} b t l\right)^{2} F^{2}+\cdots\right] F d x \\
& =\operatorname{ekl}\left(Q_{0} / h \nu\right) A\left[B_{1}+a_{1}(\theta A) B_{2}+a_{2}(\theta A)^{2} B_{3}+\cdots\right]
\end{aligned}
$$

where

$$
\begin{aligned}
\theta & =\pi \sigma^{2} b t l Q_{0} \mu / h \nu \text { and } \\
B_{n} & =\int_{0}^{w}(-1)^{n} \sinh ^{n}[\mu(x-w)+\log \sqrt{r}] \mu d x
\end{aligned}
$$

If $\gamma$ is negligible then $F=\left(Q_{0} \mu / h \nu\right) e^{-\mu x}$ and (7) becomes

$I=e k l\left(Q_{0} / h \nu\right)\left[\left(1-e^{-\mu w}\right)+\frac{1}{2} a_{1} \theta\left(1-e^{-2 \mu w}\right)+\frac{1}{3} a_{2} \theta^{2}\left(1-e^{-3 \mu w}\right)+\cdots\right](7$ 
If most of the light is absorbed in the crystal and $r$ is small it will be sufficiently accurate to correct for the light passing clear through by making $w$ infinite. With $w$ infinite $(7.1)$ becomes

$$
I=\operatorname{ekl}\left(Q_{0} / h \nu\right) f(\theta)
$$

where $f(\theta)=1+\frac{1}{2} a_{1} \theta+\frac{1}{3} a_{2} \theta^{2}+\cdots=(1 / \theta) \log J_{0}(2 i \sqrt{ } \theta)$.

The same result could be obtained by making $w$ infinite in (6).

$$
\lim _{\theta \doteq \infty} f(\theta)=\lim _{\theta \doteq \infty} J_{1}(2 i \sqrt{\theta}) / i \sqrt{\theta} J_{0}(2 i \sqrt{\theta})=0
$$

The theory thus says that, $\nu$ and $Q_{0}$ being constant, the current should drop with time as shown in Fig. 4 for $\theta$ is a linear function of the time.

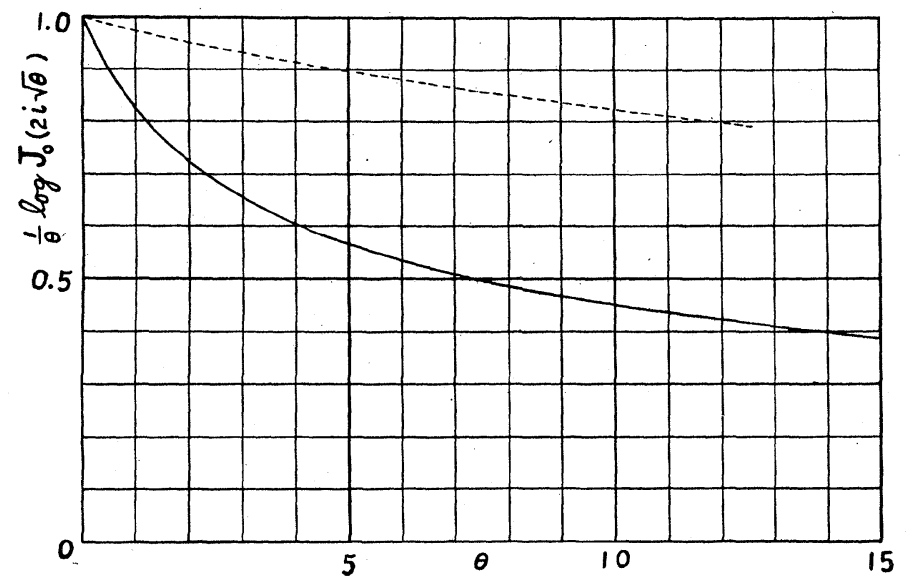

Fig. 4.

This is just what Gudden and Pohl have observed in $\mathrm{NaCl}^{\sharp}$. With intense illumination they got a drop such as shown. With weak illumination they got very little drop in the same length of time and this is to be expected from the form of $\theta$. If $Q_{0}$ is small it takes a long time for $\theta$ to become large.

If the experimental conditions were such that most of the light was absorbed in the rock salt it is permissible to compare $f(\theta)$ with the experimental curves, otherwise it would be necessary to use the more exact expression for $I$. As $b$ is not known the test of the theory will consist of seeing if the constant $b$ can be so chosen that the theoretical curve will lie along the experimental curve. The following figure shows how closely $f(\theta)$ can fit the experimental curve.

In view of the approximations introduced in deriving $f(\theta)$ and in applying it to the experiments the fit seems very good and appears to 
warrant the conclusion that recombination is sufficient to account for the decrease in the current with time and that the assumption $b=$ constant was not sensibly incorrect.

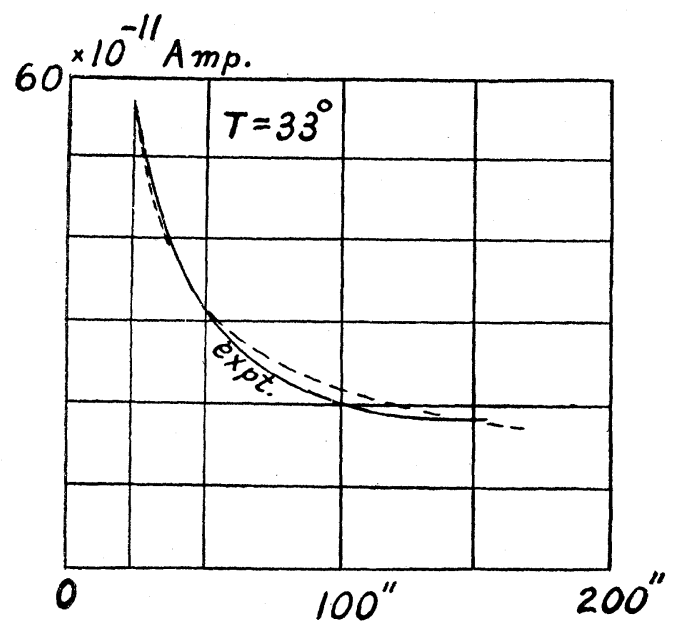

Fig. 5.

IV.

In the experiments on diamond, $\mathrm{ZnS}$ and $\mathrm{HgS}$ the light was, as shown in the accompanying figure, parallel and opposite to the electric field. The rate of production of electrons is now not a function of $x$ but of $y$

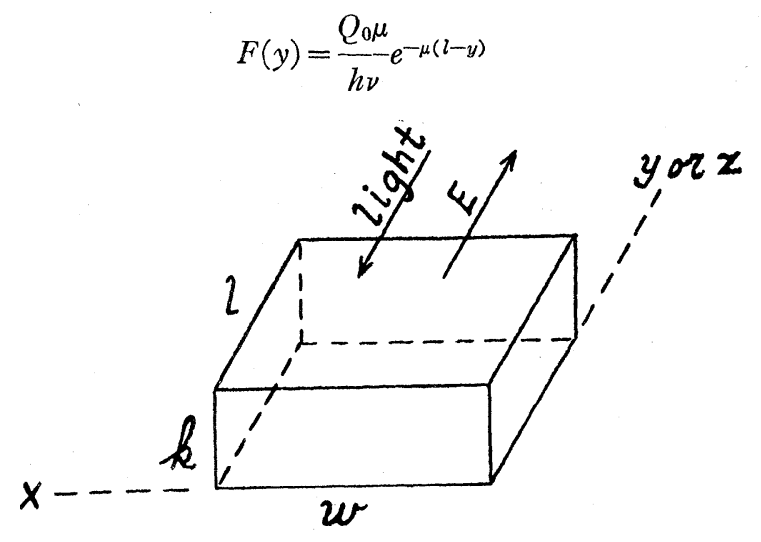

Fig. 6.

Reflection at the rear face of the crystal is neglected.

Since it was found possible and necessary to neglect the change in the density of ions while an electron is crossing the crystal, that 
simplification will now be made at the start. The total number of electrons recombining in the element of volume $k d x d y$ is got by integrating $k d x d z F(z) P(z, y, t) d y / \lambda(y)$ from $z=0$ to $z=y$ and so is

$$
k d x d y \frac{Q_{0} \mu}{h \nu} \frac{1}{\lambda(y)} \int_{0}^{y} e^{-\mu(l-z)} P(z, y, t) d z
$$

per sec. The number of electrons liberated per sec. in the same element of volume is

$$
k d x d y \frac{Q_{0} \mu}{h \nu} e^{-\mu(l-y)}
$$

and taking the difference we get, as before, the net rate of production to be

$$
k d x d y \frac{Q_{0} \mu}{h \nu}\left[e^{-\mu(l-y)}-\frac{1}{\lambda} \int_{0}^{y} e^{-\mu(l-z)} P(z, y, t) d z\right]
$$

which must equal

Therefore

$$
k d x d y \frac{\partial N}{\partial t}=k d x d y \frac{1}{\pi \sigma^{2} b} \frac{\partial}{\partial t} \frac{1}{\lambda}
$$

$$
\frac{1}{\pi \sigma^{2} b} \frac{\partial}{\partial t} \frac{1}{\lambda}=\frac{Q_{0} \mu}{h \nu} e^{-\mu l}\left[e^{\mu y}-\frac{1}{\lambda} \int_{0}^{y} e^{\mu z} P(z, y, t) d z\right]
$$

or

$$
\frac{\partial}{\partial \tau} \frac{1}{\lambda}=e^{\mu y}-\frac{1}{\lambda} \int_{0}^{y} e^{\mu z} P(z, y, t) d z
$$

where $\tau=\left(\pi \sigma^{2} b Q_{0} \mu / h \nu\right) e^{-\mu l} t$ defines $\lambda$ as a function of $t$ and $y$.

This last equation may also be written

$$
-\frac{\partial}{\partial \tau} \log \lambda=\lambda e^{\mu y}-\int_{0}^{y} e^{\mu z} P(z, y, t) d z
$$

Differentiating once with respect to $y$ we have

$$
\begin{aligned}
-\frac{\partial^{2}}{\partial y \partial \tau} \log \lambda & =e^{\mu y}\left(\frac{\partial \lambda}{\partial y}+\mu \lambda\right)-\left[e^{\mu y}-\frac{1}{\lambda} \int_{0}^{y} e^{\mu z} P(z, y, t) d z\right] \\
& =e^{\mu y}\left(\frac{\partial \lambda}{\partial y}+\mu \lambda\right)-\frac{\partial}{\partial \tau} \frac{1}{\lambda}
\end{aligned}
$$




$$
\text { Let } \begin{aligned}
e^{\mu y} & =\zeta, \frac{\partial}{\partial y}=\frac{\partial}{\partial \zeta} \frac{d \zeta}{d y}=\mu \zeta \frac{\partial}{\partial \zeta} \\
-\mu \zeta \frac{\partial^{2}}{\partial \zeta \partial \tau} \log \lambda & =\zeta\left(\mu \zeta \frac{\partial \lambda}{\partial \zeta}+\mu \lambda\right)-\frac{\partial}{\partial \tau} \frac{1}{\lambda}
\end{aligned}
$$

and get

or

$$
\begin{aligned}
\frac{\partial^{2}}{\partial \zeta \partial \tau} \log \lambda & =\frac{1}{\mu \zeta} \frac{\partial}{\partial \tau} \frac{1}{\lambda}-\left(\zeta \frac{\partial \lambda}{\partial \zeta}+\lambda\right) \\
& =\frac{\partial}{\partial \tau} \frac{1}{\mu \zeta \lambda}-\frac{\partial}{\partial \zeta}(\zeta \lambda)
\end{aligned}
$$

Placing $\lambda=e \xi, 1 / \mu \zeta=\beta, \zeta=\alpha$ this takes the standard form

$$
-\frac{\partial^{2} \xi}{\partial \zeta \partial \tau}=\frac{\partial}{\partial \zeta}\left(a e^{\xi}\right)-\frac{\partial}{\partial \tau}\left(\beta e^{-\xi}\right)
$$

of which the solution is known to be $e^{\xi}=\lambda=v / u$ where $v$ and $u$ must satisfy the equations $\alpha v=\partial u / \partial \tau$ and $\beta u=\partial v / \partial \zeta$ or $\zeta v=\partial u / \partial \tau$ and $u / \mu \zeta=\partial v / \partial \zeta$, which can be combined into the one equation

where $\tau_{1}=\tau / \mu$.

$$
v=\mu \frac{\partial^{2} v}{\partial \zeta \partial \tau} \text { or } v=\frac{\partial^{2} v}{\partial \zeta \partial \tau_{1}}
$$

If we place $e^{\mu y}=\zeta$ in the original integral equation (1) it becomes

$$
-\frac{\partial}{\partial \tau} \log \lambda=\mu \zeta-\frac{1}{\mu} \int_{1}^{\zeta} e^{-\int_{z_{1}}^{\zeta} \frac{d \zeta}{\mu \zeta \lambda}} d z_{1}
$$

Now

$$
\lambda=v / u=v / \mu \zeta \frac{\partial v}{\partial \zeta} \text { or } 1 / \mu \zeta \lambda=\partial \log v / \partial \zeta
$$

whence by substitution

$$
\frac{v}{\mu}\left(\frac{\partial v}{\partial \zeta}\right)^{-1}-\frac{1}{v} \frac{\partial v}{\partial \tau}=\frac{v}{\mu}\left(\frac{\partial v}{\partial \zeta}\right)^{-1}-\frac{1}{\mu v} \int_{1}^{\zeta} v\left(\tau, z_{1}\right) d z_{1}
$$

or

$$
\frac{\partial v}{\partial \tau_{1}}=\int_{i}^{\zeta} v\left(\tau, z_{1}\right) d z_{1}
$$


We need then a solution of $\quad v=\frac{\partial^{2} v}{\partial \zeta \partial \tau_{1}}$ that satisfies the conditions

$$
\frac{\partial v}{\partial \tau_{1}}=\int_{1}^{\zeta} v\left(\tau, z_{1}\right) d z_{1} \text { and } \lambda=\infty \text { if } t=0
$$

It has already been found that a solution of $\quad v=\frac{\partial^{2} v}{\partial \zeta \partial \tau_{1}}$ is

$$
\begin{aligned}
v & =J_{0}\left(2 i \sqrt{\left(\tau_{1}+g\right)(\zeta+h)}\right) \\
& =1+\frac{1}{(1 !)^{-2}}\left(\tau_{1}+g\right)(\zeta+h)+\frac{1}{(2 !)^{-2}}\left(\tau_{1}+g\right)^{2}(\zeta+h)^{2}+\cdots
\end{aligned}
$$

Obviously the second condition is satisfied by taking $g=0$ and the first by taking $h=-1$, we then have

$$
\begin{aligned}
& \lambda=1 / \mu \zeta \log J_{0}\left(2 i \sqrt{(\zeta-1) \tau_{1}}\right) \\
& I=\text { current }=e k \int_{0}^{w} d x \int_{0}^{l} \frac{\partial N}{\partial t} d y=e k w \int_{1}^{e^{\mu l}} \frac{\partial N}{\partial t}-\frac{d \zeta}{\mu \zeta}
\end{aligned}
$$

But $\frac{\partial N}{\partial t}=\frac{1}{\pi \sigma^{2} b} \frac{\partial}{\partial t} \frac{1}{\lambda}=\frac{1}{\pi \sigma^{2} b} \frac{\partial}{\partial t}\left[\mu \zeta \frac{\partial}{\partial \zeta} \log J_{0}\left(2 i \sqrt{(\zeta-1) \tau_{1}}\right)\right]$

therefore

$$
\begin{aligned}
I & =\frac{e k w}{\pi \sigma^{2} b} \frac{\partial}{\partial t} \int_{1}^{\mu l} \frac{\partial}{\partial \zeta} \log J_{0}\left(2 i \sqrt{(\zeta-1) \tau_{1}}\right) d \zeta \\
& =\frac{e k w}{\pi \sigma^{2} b} \frac{\partial}{\partial t} \log J_{0}\left(2 i \sqrt{\left(e^{\mu l}-1\right) \tau_{1}}\right) \\
& =e k w \frac{Q_{0}}{h \nu}\left(1-e^{-\mu l}\right) \frac{d}{d \theta_{1}} \log J_{0}\left(2 \overline{i \vee} \theta_{1}\right)
\end{aligned}
$$

where

$$
\theta_{1}=\left(1-e^{-\mu l}\right) \pi \sigma^{2} b t Q_{0} / h \nu
$$

or

$$
\begin{aligned}
I & =e k w \frac{Q_{0}}{h \nu}\left(1-e^{-\mu l}\right) \frac{J_{0}\left(2 i \sqrt{\theta_{1}}\right)}{i \sqrt{ } \theta_{1} J_{0}\left(2 i \sqrt{ } \theta_{1}\right)} \\
\lim _{t=\infty} I & =\lim _{\theta_{1}=\infty} I=0
\end{aligned}
$$


This shows how recombination should make the current drop with time when the light is parallel and opposite to the electric field.

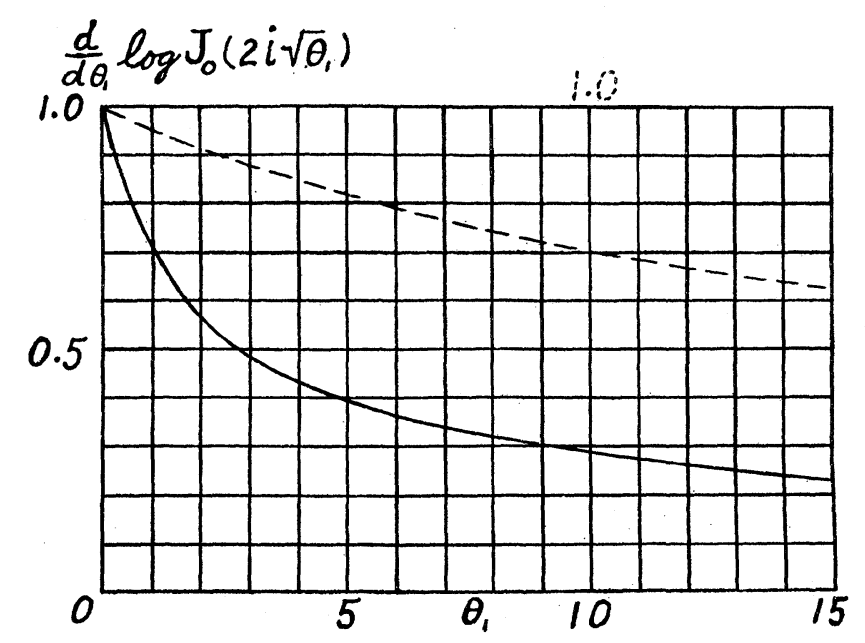

Fig. 7.

The quantity of electricity passing to the anode between the times $t=0$ and $t=t$ is

$$
\begin{aligned}
C & =\int_{0}^{t} I d t=\int_{0}^{t} \frac{e k w}{\pi \sigma^{2} b} \frac{\partial}{\partial t} \log J_{0}\left(2 i \sqrt{\left(e^{\mu l}-1\right) \tau_{1}}\right) d t \\
& =\left(e k w / \pi \sigma^{2} b\right) \log J_{0}\left(2 i \sqrt{\left(1-e^{-\mu l}\right) \pi} \overline{\sigma^{2} b t Q_{0} / h \nu}\right) \\
& =e k w\left(Q_{0} / h \nu\right)\left(1-e^{-\mu l}\right) t\left(1 / \theta_{1}\right) \log J_{0}\left(2 i \sqrt{\theta_{1}}\right)
\end{aligned}
$$

For short observation periods ( $t$ small) the quantity $c$ is thus seen to be proportional to $t$ for $f(0)=1$. This is in agreement with the measurements. of Gudden and Pohl. ${ }^{1}$

If recombination is neglected, i. e. if $f\left(\theta_{1}\right)$ is made equal to one, $C=e k w Q_{0} t / h \nu$ which is a straight line when plotted aginst $1 / \nu$. The factor $\left(1-e^{-\mu l}\right)$ is left off to correct for the light that goes clear through and is lost. It is this $C$ which Gudden and Pohl measured in their experiments on diamond, $\mathrm{HgS}$, and $\mathrm{ZnS}$.

The dotted curve in Fig. 8 gives the results of the experiments on $\mathrm{ZnS}$ in terms of $C / Q_{0}$. The dashed straight line is the $C / Q_{0}$ which would have been obtained had every $h \nu$ liberated one electron which also reached the anode. It was located by multiplying the ordinate at $405 \mu \mu$ by $7 / 6.55$. Neglecting recombination the experimental value, of $h$ is here $7 \times 10^{-27}$ instead of $6.55 \times 10^{-27}$. The difference is said to 
be within the experimental error. The full curve is the $C / Q_{0}$ given by Eq. (3). It was calculated with the aid of the curves given by Gudden and Pohl ${ }^{1}$ for $\mu$ in $\mathrm{ZnS}$, taking $l=.6 \mathrm{~mm}$ and so choosing $b$ as to make the two curves coincide at $\lambda=400 \mu \mu$. It represents then the "Ausbeute" to be expected if recombination were the only phenomenon tending to reduce the current, and provided the phenomenon other than recombination which causes the large drop at $350 \mu \mu$ is inoperative at and beyond $400 \mu \mu$. Judging from the shape of the dotted curve this is the case.

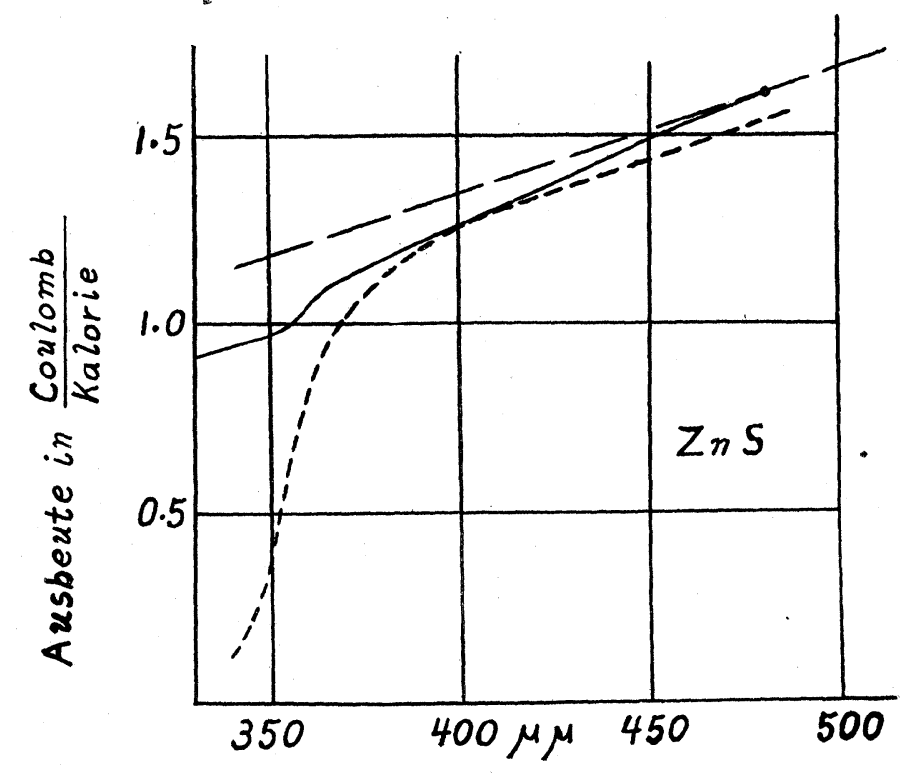

Fig. 8.

The peculiar drop is such as would be caused by a rapid increase in $b$ between 400 and $340 \mu \mu$; this does not seem at all likely. If it is caused by initial recombination or some other reason amounting to the same thing it is equivalent to a decrease in $Q_{0}$, and we must have $Q_{0}=Q_{0}(\nu)$. This does not affect the validity of equations (2) and (3) because the integrations were only with respect to space and time.

The effect of the unknown process causing the peculiar drop in current when the light is most strongly absorbed is thus seen to be less than it would seem to be were recombination neglected. By determining $b$ where the effect is inappreciable we see how big the effect is at the shorter wave-lengths.

Norman Bridge Laboratory of Physics, California Institute of Technology, March 8, 1926. 